\title{
Modulated waves in a periodically driven annular cavity
}

\author{
H. M. BLACKBUR $N^{1} \uparrow$ AND J. M. LOPEZ \\ ${ }^{1}$ Department of Mechanical and Aerospace Engineering, Monash University, Vic 3800, Australia, \\ ${ }^{2}$ School of Mathematical and Statistical Sciences, Arizona State University, Tempe, AZ 85287, USA \\ (Received 16 April 2010; revised 22 August 2010; accepted 22 August 2010; \\ first published online 25 November 2010)
}

Time-periodic flows with spatio-temporal symmetry $Z_{2} \times O(2)$ - invariance in the spanwise direction generating the $O(2)$ symmetry group and a half-period-reflection symmetry in the streamwise direction generating a spatio-temporal $Z_{2}$ symmetry group - are of interest largely because this is the symmetry group of periodic laminar two-dimensional wakes of symmetric bodies. Such flows are the base states for various three-dimensional instabilities; the periodically shedding two-dimensional circular cylinder wake with three-dimensional modes A and B being the generic example. However, it is not easy to physically realize the ideal flows owing to the presence of end effects and finite spanwise geometries. Flows past rings are sometimes advanced as providing a relevant idealization, but in fact these have symmetry group $O(2)$ and only approach $Z_{2} \times O(2)$ symmetry in the infinite aspect ratio limit. The present work examines physically realizable periodically driven annular cavity flows that possess $Z_{2} \times O(2)$ spatio-temporal symmetry. The flows have three distinct codimension-1 instabilities: two synchronous modes (A and $\mathrm{B})$, and two manifestations of a quasi-periodic (QP) mode, either as modulated standing waves or modulated travelling waves. It is found that the curvature of the system can determine which of these modes is the first to become unstable with increasing Reynolds number, and that even in the nonlinear regime near onset of three-dimensional instabilities the dynamics are dominated by mixed modes with complicated spatio-temporal structure. Supplementary movies illustrating the spatio-temporal dynamics are available at journals.cambridge.org/flm.

Key words: bifurcation, pattern formation, vortex streets

\section{Introduction}

Typically, spatial patterns in non-equilibrium hydrodynamic systems have been classified according to the linear instabilities of an infinite spatially uniform system, or at least a system which is infinite in a direction in which a pattern forms, as a control parameter exceeds a critical value (Cross \& Hohenberg 1993). While this approach has worked extremely well for stationary patterns (such as rolls in Rayleigh-Bénard convection and Taylor vortex cells), when the pattern propagates in the model problem, its physical manifestation is often compromised by the patternforming direction not being truly translation-invariant. One of the major problems 
with this modelling approach is that for a system with an infinite direction the usual linear stability analysis in terms of normal modes cannot be performed directly, as the associated spectrum is continuous. To remedy this, an arbitrary periodicity is imposed in the infinite direction, which renders the spectrum discrete and allows for a normal mode analysis, but then the resultant pattern depends on the arbitrarily imposed periodicity. When the pattern-forming direction is physically periodic, e.g. an angular direction, then the modal approach is directly applicable.

Also, there has been much interest in pattern formation from non-trivial states. A classic example is the transition to three-dimensional flow from the two-dimensional periodic wake of bluff bodies; the von Kármán vortex street wake of a circular cylinder being the prototypical case. It was early recognized that weak three-dimensional effects induced by the cylinder ends are important, even for very large length-todiameter aspect ratio cylinders, in nominally two-dimensional low-Reynolds-number wakes (Williamson 1989). Nevertheless, the nominally two-dimensional experiments of Williamson (1996) identified two synchronous three-dimensional modes (modes A and B) which were captured by the Floquet analysis of the two-dimensional cylinder wake flow (Barkley \& Henderson 1996; Henderson \& Barkley 1996). Blackburn \& Lopez (2003a) showed that there is a third pattern-forming instability of the twodimensional wake, first alluded to in Barkley \& Henderson (1996), which results in a quasi-periodic three-dimensional flow, mode QP (quasi-periodic solutions possess two, generally incommensurate, distinct temporal periods). Other flows with the same symmetries (invariance in the spanwise direction generating the $O(2)$ symmetry group and a half-period-reflection symmetry in the streamwise direction generating a spatio-temporal $Z_{2}$ symmetry group) have also been found to produce synchronous three-dimensional patterns with the same characteristics as those of the cylinder wake (Meiburg \& Lasheras 1988; Lasheras \& Meiburg 1990; Julien, Lasheras \& Chomaz 2003; Vogel, Hirsa \& Lopez 2003). The physical mechanisms underlying various instability modes, especially synchronous ones, have been the subject of ongoing debate, as outlined by Blackburn \& Lopez (2003b) and references therein. However, our main focus here is on the dynamics of quasi-periodic modes in these systems.

Blackburn \& Lopez (2003b) analysed the three-dimensional instabilities of a periodically driven infinite rectangular cavity flow. As for the cylinder wake flows, the cavity flow has two synchronous modes (named A and B as a result of visual similarities to the equivalent cylinder wake modes), in addition to a quasi-periodic mode. The symmetry group of the problem is spatio-temporal $Z_{2} \times O(2)$, just as in the periodically shedding circular cylinder wake problem, but the driven cavity is a nonautonomous flow, and this provides one of the attractions of adopting such a system as a vehicle with which to study bifurcations in flows with this symmetry. Different combinations of the amplitude and frequency of motion of the oscillating wall lead to variation in which the three types of instability modes can be made to bifurcate directly from the base state - the system has two dynamic control parameters which allow this freedom of choice. In contrast, autonomous time-periodic flows, such as two-dimensional circular and square cylinder wakes, typically possess only one control parameter (the free-stream velocity as characterized by the Reynolds number), and consequently, the three modes bifurcate from the base state sequentially as this single control parameter is varied.

Blackburn, Marques \& Lopez (2005) explored the relationship between the threedimensional instabilities of the idealized two-dimensional cylinder wake and driven cavity flows, and Marques, Lopez \& Blackburn (2004) provided a detailed centre manifold and normal form analysis of the bifurcations involved. An experimental 
test of the theoretical predictions from Marques et al. (2004) was presented in Leung et al. (2005), where the physical periodically driven cavity problem with finite span had $Z_{2} \times Z_{2}$ spatio-temporal symmetry (the spanwise translation/reflection invariance $O(2)$ in the model problem being reduced to a spanwise reflection $Z_{2}$ in the physical experiment). The synchronous modes A and B were captured well (as they are in the cylinder wake experiments), but the QP mode, which the model predicted to be a (modulated) travelling wave state, was not. A quasi-periodic state was observed experimentally for the parameter ranges predicted, with its two frequencies and the spanwise wavelength of the three-dimensional instability in good agreement with the model predictions, but its spatio-temporal form was not that of a (modulated) travelling wave.

In this paper, we examine a continuous deformation of the idealized planar driven cavity flow such that the infinite spanwise direction becomes a periodic angular direction. The transformation preserves the $Z_{2} \times O(2)$ symmetry of the original system owing to the fact that the direction in which the flow is driven through oscillation lies normal to the direction of distortion. The motivation for this was to identify parameter regimes where the (modulated) travelling waves predicted by Blackburn \& Lopez (2003b) and Marques et al. (2004) continue to be the primary three-dimensional mode of instability in the modified, but now physically realizable, annular problem. In our driven cavity problem, the planar version has a well-defined supercritical bifurcation for the onset of (modulated) travelling waves, and we find that this mode of instability can be continued into the annular version by smooth variations in curvature. The ability to produce primary bifurcations to synchronous modes A and B is also retained in this system, but here the main focus is on control parameter combinations that determine mode QP to be the primary instability.

We note that other physically realizable fluid-mechanical systems with $Z_{2} \times O(2)$ symmetry are possible. One simple case is obtained by removing the inner annulus of the present geometry, so that there is a cylindrical cavity with fixed endwalls and only an axially sliding oscillatory outer wall. The annular geometry adopted in the present work, however, allows the original rectangular geometry studied in Blackburn $\&$ Lopez (2003b) to be obtained in the zero-curvature limit. Before leaving this point, we also note that the same type of deformation (infinite planar direction deformed into a circular direction) may not preserve the original symmetry in other systems if the direction of oscillation is not orthogonal to the direction of deformation. An example that has received considerable attention is flow past rings of circular cross-section, taken as a model of an infinite circular cylinder (Leweke \& Provansal 1995; Sheard, Thompson \& Hourigan 2003). When the free-stream flow is directed along the ring axis and Reynolds numbers are high enough for vortex shedding to occur, the instability generates an oscillatory velocity component normal to the axis, which is in the direction of deformation. As a result, deformation destroys the $Z_{2}$ symmetry group characteristic of the two-dimensional von Kármán wake and central to the class of flows treated here. Breaking this symmetry can change the nature of three-dimensional bifurcations that are possible from the two-dimensional basic state. Of particular significance in relation to the present work is the fact that subharmonic modes which can arise in systems with broken $Z_{2}$ symmetry (as shown by Sheard et al. 2003, Carmo et al. 2008, Sheard, Fitzgerald \& Ryan 2009, respectively, for wakes of rings, of a staggered pair of circular cylinders, and of a rotated square cylinder) are suppressed as generic bifurcations in symmetric systems. This outcome is a direct consequence of $Z_{2}$ spatio-temporal symmetry, as originally explained for simple systems by Swift \& Wiesenfeld (1984), and in depth for $Z_{2} \times O(2)$ 
(a)

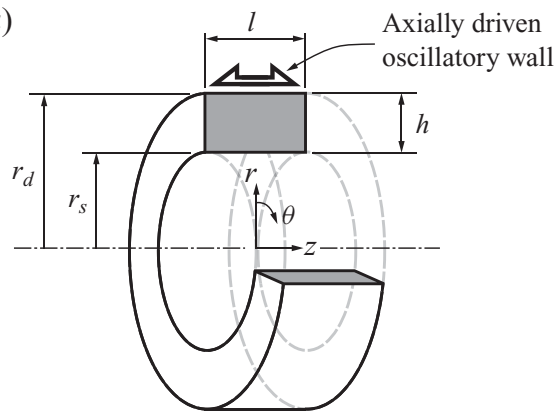

(b)

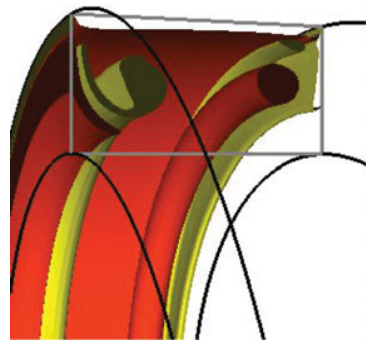

$t=\tau / 2$
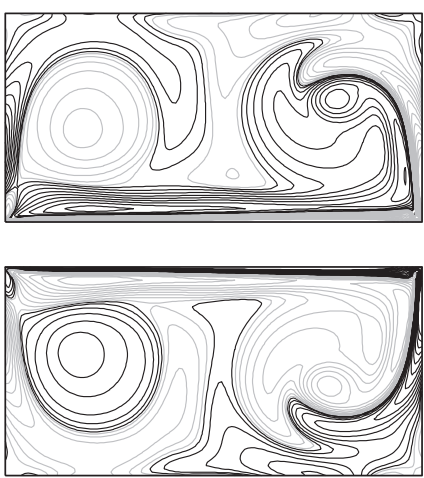

Figure 1. (Colour online) (a) Schematic of the flow geometry (the oscillatory outer wall case is shown). (b) Cutaway perspective view of a typical basic state visualized as \pm isosurfaces of azimuthal vorticity of equal magnitude at one instant in the wall motion cycle, for $\Psi=4 / 3$, $S t=100$ and $R e=1200$ (see supplementary movie 1 for an animation). Snapshot contours of base flow azimuthal vorticity (black, positive; grey, negative) half a period apart for $(c)$ $\Psi=9 / 10$ (inner-wall-driven) and (d) $\Psi=10 / 9$ (outer-wall-driven), both at $S t=100, R e=1200$.

systems by Marques et al. (2004). Recently, Blackburn \& Sheard (2010) demonstrated the connection: a finite amount of deformation, which breaks the $Z_{2}$ symmetry to leave only $O(2)$, typically transforms a quasi-periodic instability mode into a pair of subharmonic instability modes.

\section{Problem definition, dimensionless groups and symmetries}

Consider the flow in a finite annular channel driven by the harmonic oscillation in the axial direction $z$ of either the inner or the outer cylindrical wall. The radius of the driven wall is $r_{d}$ and that of the stationary cylindrical wall is $r_{s}$; their ratio $\Psi=r_{d} / r_{s}$ is greater or less than unity depending on which wall oscillates. The infinite rectangular channel with zero curvature (Blackburn \& Lopez 2003b) is recovered in the limit $\Psi \rightarrow 1$. The geometry and coordinate system used for the problem are illustrated in figure 1. The rectangular cross-section of the annulus has radial gap, $h$, and axial length, $l$.

Taking the radial gap, $h$, as the length scale and the maximum wall velocity, $W_{\max }$, as a velocity scale (so that the time scale is $h / W_{\max }$ ), the non-dimensional governing 
equations and boundary conditions are:

$$
\begin{array}{ll}
\text { Navier-Stokes } & \partial_{t} \boldsymbol{u}+\boldsymbol{u} \cdot \nabla \boldsymbol{u}=-\nabla P+R e^{-1} \nabla^{2} \boldsymbol{u}, \\
\text { incompressibility } & \nabla \cdot \boldsymbol{u}=0, \\
\text { boundary conditions } & \left\{\begin{array}{l}
\boldsymbol{u}(r, \theta, \pm \Gamma / 2)=\mathbf{0}, \\
\boldsymbol{u}\left(r_{s}, \theta, z\right)=\mathbf{0}, \\
\boldsymbol{u}\left(r_{d}, \theta, z\right)=[0,0, \sin (2 \pi t / \tau)],
\end{array}\right.
\end{array}
$$

where $\boldsymbol{u}=(u, v, w)$ is the velocity field in polar coordinates $(r, \theta, z)$ and $P$ is the kinematic pressure. The vorticity is $\nabla \times \boldsymbol{u}=(\xi, \eta, \zeta)=\left(r^{-1} \partial_{\theta} w-\partial_{z} v, \partial_{z} u-\right.$ $\left.\partial_{r} w, r^{-1} \partial_{r}(r v)-r^{-1} \partial_{\theta} u\right)$.

There are four governing parameters:

$$
\begin{array}{rlrl} 
& \text { the length-to-gap aspect ratio } & \Gamma & =l / h, \\
\Psi & =r_{d} / r_{s}, \\
& \text { the radius ratio } & R e & =W_{\max } h / v, \\
& \text { the Reynolds number } & S t & =h^{2} / T \nu,
\end{array}
$$

where $T$ is the driven wall's dimensional forcing period; the dimensionless values of wall motion period and maximum displacement are $\tau \equiv R e / S t$ and $\tau / 2 \pi$, respectively.

The system $(2.1 a)-(2.1 c)$ is invariant to a number of symmetry operations: rotations about the axis, $R_{\alpha}$, whose action on velocity and vorticity is

$$
\begin{aligned}
R_{\alpha}(u, v, w)(r, \theta, z) & =(u, v, w)(r, \theta+\alpha, z), \\
R_{\alpha}(\xi, \eta, \zeta)(r, \theta, z) & =(\xi, \eta, \zeta)(r, \theta+\alpha, z),
\end{aligned}
$$

reflections about any meridional plane, $K_{\theta}$, whose action is

$$
\begin{aligned}
K_{\theta}(u, v, w)(r, \theta, z) & =(u,-v, w)(r,-\theta, z), \\
K_{\theta}(\xi, \eta, \zeta)(r, \theta, z) & =(-\xi, \eta,-\zeta)(r,-\theta, z),
\end{aligned}
$$

and a spatio-temporal symmetry $H$, composed of a reflection about $z=0$ and a temporal evolution of a half-period, whose action is

$$
\begin{aligned}
H(u, v, w)(r, \theta, z, t) & =(u, v,-w)(r, \theta,-z, t+\tau / 2), \\
H(\xi, \eta, \zeta)(r, \theta, z, t) & =(-\xi,-\eta, \zeta)(r, \theta,-z, t+\tau / 2) .
\end{aligned}
$$

The symmetries $R_{\alpha}$ and $K_{\theta}$ do not commute and together they generate the spatial group $O(2) ; H$ generates a $Z_{2}$ group which commutes with the $O(2)$ group, and so the complete symmetry group of the system is $Z_{2} \times O(2)$. As discussed in $\S 1$, other fluid-mechanical systems such as axisymmetric vortex-street ring wakes (e.g. Leweke $\&$ Provansal 1995) that may initially seem closely related to the present case, allow the spatio-temporal symmetry $H$ to be obtained only in the zero-curvature limit. Consequently, for any finite deformation/curvature, such flows have $O(2)$ symmetry group, generated by $R_{\alpha}$ and $K_{\theta}$, as opposed to $Z_{2} \times O(2)$.

In the present study, as in Blackburn \& Lopez (2003b), the aspect ratio is fixed at $\Gamma=2$, while the remaining geometric parameter, radius ratio $\Psi$, which gives a measure of the curvature in the problem, is varied. This generalizes the previous work, which corresponded to the limit $\Psi=1$. Note that as $\Psi \rightarrow 1$ from below, there is a switch from inner cylindrical wall to outer cylindrical wall oscillations. At $\Psi=1$ the system is invariant to which wall oscillates. Since our original motivation lay in studying the modulated travelling wave states, we also fixed the Stokes number, $S t=100$, as it was 
established in the Cartesian case that this value was near the middle of the range of Stokes numbers $(S t \in[87.5,132])$ over which the quasi-periodic modes were first to bifurcate from the two-dimensional basic states. In the Cartesian case at $S t=100$, the quasi-periodic mode bifurcates from the two-dimensional base state at $\operatorname{Re}_{c}=1212$, while the synchronous mode $\mathrm{A}$ is the second to bifurcate at $R e_{c}=1371$. The other synchronous mode, B, bifurcates at a considerably higher value, which we have not conclusively established but is at least $R e=1500$ for $S t=100$.

Thus, of the four control parameters shown in (2.2), we fix $\Gamma=2$ and $S t=100$, and examine the effect of variations in $\Psi$ and $R e$, with focus principally on quasiperiodic instability modes. A further point regarding the nomenclature for these quasi-periodic modes is in order here. When considering linear instability (e.g. in $\S 5)$, we generally refer simply to mode $\mathrm{QP}$, which encompasses both modulated azimuthally travelling wave (TW) and modulated standing wave (SW) states. Of the three symmetries possessed by the basic state, TW states break $K_{\theta}$, while SW states (symmetric combinations of TWs) break $R_{\alpha}$. Both TW and SW states bifurcate linearly from the basic state at the same point in parameter space and share the same azimuthal wavenumber. The introduction of nonlinearity favours the stability of one of the SW/TW pair over the other (Crawford \& Knobloch 1991), and in this problem it is found that TW is the favoured state. When nonlinear solutions are considered in $\S \S 6-7$, we typically refer to TW or SW states as instances of mode QP.

\section{Methodology}

The computational methods employed were previously described and applied in Blackburn (2002), Blackburn \& Lopez (2002, 2003a,b), Blackburn \& Sherwin (2004), Blackburn et al. (2005) and Barkley, Blackburn \& Sherwin (2008), and so only a brief overview is presented here.

\subsection{Discretization}

The underlying discretization is achieved using nodal spectral elements for the $(r, z)$ meridional semi-plane shown in figure 1, coupled with Fourier expansions in azimuth, or, in the limit $\Psi=1$, in the out-of-plane direction. The same discretization is used to compute base flows, linear stability analysis and direct numerical simulation (DNS). The previous study of instability in the Cartesian geometry (Blackburn \& Lopez $2003 \mathrm{~b}$ ) used 108 spectral elements with nodal seventh-order-tensor-product spectral element shape functions and presented a detailed convergence study to establish that this provided adequate spatial resolution in the parameter regimes studied here. For the present work, meshes with 176 elements and at least seventh-order shape functions were adopted. Time advancement is second-order, with 3096 steps (or more) per wall period. Again, this exceeds the resolution of the previous study.

The mesh is mapped as required by reflection about the mean radius to place maximum resolution near the driven wall depending on whether this motion occurs at the inner or outer radius. For computation of base flows and three-dimensional DNS, the tangential wall velocity is brought to zero near the intersection with the stationary walls using

$$
\boldsymbol{u}\left(r_{d} / h, \theta, z\right)=\left[1-\exp \left(-200(1+z)^{4}\right)\right]\left[1-\exp \left(-200(1-z)^{4}\right)\right][0,0, \sin (2 \pi t / \tau)],
$$


to provide a smooth regularization of the boundary condition singularity that occurs in the corners. This regularization was also used in the earlier Cartesian-geometry study.

\subsection{Floquet analysis}

The global linear asymptotic stability of the $\tau$-periodic base flow is studied using Floquet analysis; the spatio-temporal symmetry $H(2.3 e)$ of the base flow is incorporated into the analysis by basing this on a half-period flip map instead of the full-period Poincaré map (see Blackburn et al. 2005, for details). The Krylov-type numerical method is based on time-stepping the linearized Navier-Stokes operator, as described in Tuckerman \& Barkley (2000), Barkley et al. (2008), and used e.g. in Blackburn \& Lopez (2003b). Base flows required for Floquet analysis are precomputed as limit states of two-dimensional DNS, and reconstructed within the stability analysis code via Fourier interpolation in time from a number of time slices. For the present problem we have typically employed 128 time slices, double the number used in Blackburn \& Lopez (2003b).

Floquet solutions are of the form $\boldsymbol{u}^{\prime}(t)=\exp (\sigma t) \tilde{\boldsymbol{u}}(r, \theta, z, t \bmod \tau)+$ c.c., where in general both the Floquet exponents $\sigma$ and the $\tau$-periodic eigenfunctions $\tilde{\boldsymbol{u}}$ are either real or occur in complex-conjugate pairs. Floquet modes are unstable if the real part of the exponent $\sigma$ is positive or equivalently if the Floquet multiplier $\mu=\exp (\sigma \tau)$ lies outside the unit circle in the complex plane. Note that the synchronous modes A and B have real Floquet multipliers, while mode QP has complex-conjugate-pair multipliers. For the synchronous modes A and B the Floquet multipliers for the Poincare map are both positive; however, since mode A breaks while mode B preserves $H$-symmetry, the Floquet multipliers based on the half-period flip map for mode B are positive, while those for mode A are negative (for details see Marques et al. 2004, Blackburn et al. 2005). In contrast, a subharmonic mode would correspond to a negative real multiplier of the Poincare map, and as described in $\S 1$, such a mode does not arise in the present (or any other $Z_{2} \times O(2)$-symmetric) system.

\section{Basic state}

The basic state is an axisymmetric time-periodic flow, synchronous with the forcing, which is invariant under $R_{\alpha}, K_{\theta}$ and $H$. Its features have been previously described for the planar case $\Psi=1$ (Vogel et al. 2003; Blackburn \& Lopez 2003b), and these are not qualitatively affected by curvature for the range of $\Psi$ we consider here, so we provide only a brief description of the salient features. Axial oscillations of either the inner or outer cylinder produce periodic Stokes-type layers on the oscillating wall, which separate owing to the presence of the two endwalls to form rollers alternatively on each end as the stroke of the oscillating cylinder changes. For small forcing frequencies, there is sufficient time at low enough forcing amplitudes for these rollers to dissipate during part of the cycle, whereas for large frequencies there is a balance between (slow) dissipation and (fast) boundary-layer forcing so that rollers persist at both ends throughout the whole forcing cycle. In this paper, the main focus is on the regime where, for the planar case $\Psi=1$, the primary instability of the basic state is to TW, and this occurs for intermediate frequencies $(S t \sim 100)$. Figure 1 and supplementary movie 1 show typical basic states in this regime. 


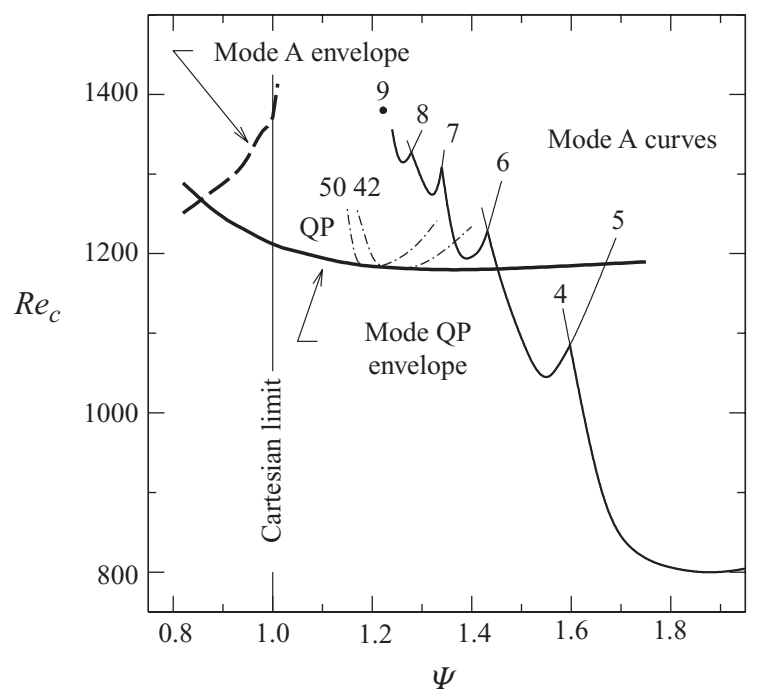

FIGURE 2. Critical Reynolds numbers as a function of radius ratio $\Psi=r_{d} / r_{s}$ for linear onset of modes A and QP at $S t=100$. Individual marginal stability curves (thin solid and chain lines) are labelled by their integer azimuthal wavenumbers. Values of $\Psi>1$ correspond to the outer cylindrical wall being driven, while $\Psi<1$ correspond to driving at the inner wall; see figure 1. Envelopes of marginal stability curves are indicated for mode QP (thick solid line) across the range $\Psi \in(0.8,1.65)$ and for mode $\mathrm{A}$ at values near and below $\Psi=1$ (dashed line). For $\Psi>1$ the mode A stability envelope is drawn as the lower-bound union of the individual curves.

\section{Linear stability analysis results}

The findings of the linear stability analysis for $S t=100, \Gamma=2$ are summarized in figures 2 and 3. As noted in $\S 2$, the two previously identified critical Reynolds numbers in the Cartesian limit are $R e_{c}=1212$ for mode QP and $R e_{c}=1371$ for mode A. These three-dimensional instabilities are associated with spanwise wavelengths $\lambda=0.739$ and $\lambda=3.81$, with corresponding spanwise wavenumbers $\beta=2 \pi / \lambda=8.5$ and 1.65 , respectively (for non-dimensional wavenumbers, we use $\beta$, which is real for the Cartesian case $\Psi=1$, and $k$, which is integer, for the annular cases with $\Psi \neq 1$ ). At this Stokes number the critical $R e$ for mode B lies above the upper limit of figure 2. Discussion of the physical characteristics of modes A and QP is deferred to $\S 6$; the focus of this section is on marginal stability Reynolds numbers, azimuthal wavenumbers and wavelengths.

Mode QP has a family of marginal stability curves, one curve for each integer azimuthal wavenumber $k$; two such curves are drawn (dot-dash style) for $k=42$ and 50 in figure 2. Each curve has a single minimum, and as $\Psi \rightarrow 1$ in either direction, $k$ values associated with these minima approach infinity and the curves become densely packed. To simplify representation, only an envelope of the minima has been drawn in the figure (thick solid line). This envelope itself has a minimum critical $R e \approx 1180$ at $\Psi \approx 1.33$.

For the longer-wavelength mode A, there is another family of marginal stability curves. At any value of $\Psi$, the values of $k$ for the mode A curves are considerably less than for the mode QP curves, corresponding to the larger wavelengths for mode A instabilities relative to mode QP. On either side of the Cartesian limit, $\Psi=1$, the minimum marginal stability $R e$ for each curve falls with increasing $|\Psi-1|$. For $\Psi<1$, only the envelope of the mode A curves is shown. The associated critical $R e$ drop rapidly with $\Psi$ and fall below those for the mode QP envelope at $\Psi \approx 0.85$. 


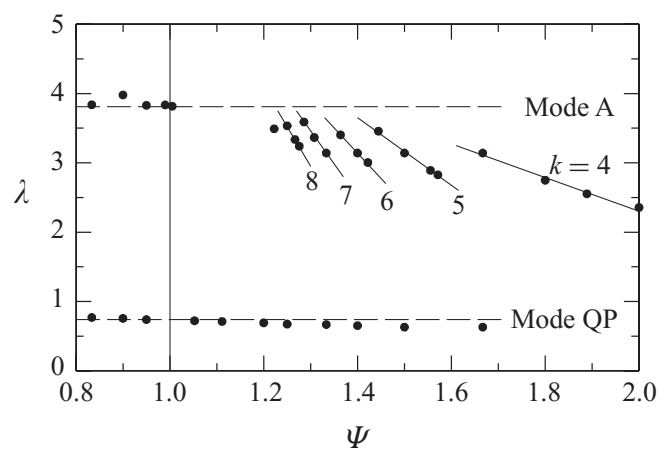

FIGURE 3. Most amplified Floquet modal azimuthal wavelength estimated at the mean radius as a function of $\Psi$. Dashed lines represent values for modes A and QP in the Cartesian geometry (Blackburn \& Lopez 2003b).

However, as $\Psi$ increases just beyond unity, the critical Re for mode A rise rapidly, and for $\Psi \in[1.02,1.2]$ the critical Re lie above the upper limit of figure 2. For $\Psi>1.2$, there is a family of critical curves whose $R e$ minima reduce with increasing $\Psi$ and the corresponding values of $k$ also fall, this being a result of only being able to fit a smaller finite number of wavelengths within the annulus. In this case the stability envelope is the lower-bound union of the individual curves, and takes on a distinctly scalloped shape. For $k \leqslant 5$, the critical $R e$ for mode A drop well below those for mode QP. This occurs for $\Psi \gtrsim 1.45$.

The azimuthal wavelengths of the most-amplified modes are related to those in the Cartesian geometry. Using the dimensionless mean radius $\bar{r}=\left(r_{d}+r_{s}\right) / 2 h$, we define the wavelength $\lambda=\bar{r} \Delta \theta=2 \pi \bar{r} / k$, where $\Delta \theta$ is the angular extent of the instability mode and $k$ is the corresponding wavenumber. This leads to the prediction

$$
\lambda=\frac{\Psi+1}{|\Psi-1|} \frac{\pi}{k}
$$

Using the most amplified wavenumbers obtained from the Floquet analysis (figure 2), the wavelengths based on the mean radius are computed from (5.1) and plotted in figure 3. The wavelengths in the annular geometries correspond quite well to those in the Cartesian case, especially for mode QP. For mode A the agreement is best near $\Psi=1$; for larger $\Psi$ values, only a small number of wavelengths fit within the annulus, and quantization effects drive the wavelengths away from the value predicted based on the mean radius and (5.1).

At moderate values of $\Psi$ near unity (in the range of 0.85-1.4, approximately), mode QP becomes linearly unstable at lower Re than does mode A and the most amplified wavelengths for the two modes are quite distinct. These observations suggest that one might observe quasi-periodic solutions in the full-circle nonlinear system (i.e. in DNS and physical experiments) for values of $\Psi$ moderately close to unity, and for Reynolds numbers slightly above the mode QP instability envelope curve. Prior to dealing with solutions in the full annulus, we first examine behaviour of the two modes in subspaces of restricted wavelength.

\section{Nonlinear behaviour with subspace restriction}

The linear stability analysis of the basic states provides the most critical wavenumbers for instability of modes A and QP. In this section, nonlinear behaviour in subspaces restricted to the critical wavenumber for each mode is investigated. The 
initial conditions for DNS consist of a basic state at Re a few per cent above the critical value (computed in an axisymmetric subspace) to which a small multiple of an unstable mode shape as derived from Floquet analysis is added. This combination is then evolved via DNS to an asymptotic/saturated state whose equilibrium is determined by nonlinear effects. Generally, close to criticality, the shape of the nonaxisymmetric component is quite close to that of the linear instability mode, while the axisymmetric component is close to the base flow. Typically, four azimuthal Fourier modes (eight planes of real data) are used, which is sufficient to accurately describe these weakly nonlinear states (for purposes of visualization, we project the solutions onto a higher number of Fourier modes).

\subsection{Descriptions of modes $Q P$ and $A$ for variable $\Psi$}

The instability leading to the quasi-periodic modes involves complex-conjugate-pair Floquet multipliers and mode shapes that break some symmetries of the base state, with the imaginary part of the critical multipliers providing a second frequency which generically is incommensurate with the base state frequency. The quasi-periodic modes come in different variants, either as TWs propagating in the azimuthal direction (either positive or negative $\theta$-direction), or a symmetric combination of these leading to an SW. Since the temporal variation of the base flow modulates these instabilities, the resulting flows are either modulated travelling waves or standing waves, but for brevity we will simply refer to them as TW or SW.

As shown in Marques et al. (2004), the onset of TW or SW states in the Floquet problem is analogous to the $O(2)$-symmetry breaking Hopf bifurcation (Crawford \& Knobloch 1991), and as in that case, typically the most energetic of the TW or SW states is stable, while the other is unstable. A pure solution of either SW or TW type can, however, be initiated with a suitable choice of initial conditions and can be maintained indefinitely under nonlinear evolution with a symmetry-respecting code. For example, to obtain an SW state the initial perturbation is constrained to have reflection symmetry in azimuth and the system will retain this symmetry under evolution by the Navier-Stokes equations. TWs come in conjugate pairs with mean travel in either the positive or negative azimuthal direction. The sense of travel depends on an arbitrary choice of sign of the azimuthal velocity component of the initial velocity perturbation. One TW solution can be generated from the other by a reflection of the azimuthal velocity component in any $(r, z)$ plane (equivalently by complex conjugation of this velocity component in Fourier space).

Figure 4 shows vorticity isosurfaces of the TW and SW saturated states at $\Psi=4 / 3$, $k=33$ and $R e=1200\left(R e_{c}=1180\right.$ for marginal stability). With $\Psi>1$, the outer wall of the annulus is driven and the view is taken outwards from the axis. Note that since this is a restricted subspace with $k=33$, only $1 / 33 \mathrm{rd}$ of the full annular cavity is represented. At this slightly supercritical $R e$, the azimuthal vorticity isosurfaces are only very slightly distorted away from axisymmetry; the radial vorticity (which is zero for axisymmetric flow) isosurfaces illustrate the nature of the three-dimensional disturbances, consisting of braids connecting the predominantly axisymmetric rollers. The views shown in the figure are snapshots strobed once every forcing period $\tau$. We note the general similarity and dynamic behaviour of the TW and SW states of figure 4 to those for equivalent states in the Cartesian geometry at $S t=100$ and $R e=1225$ (see Blackburn \& Lopez 2003b, figures 14 and 15).

For the TW case, the solution is identical in each snapshot, save for a uniform azimuthal translation in the $+\theta$-direction, although there is a continuous temporal modulation when the view is not strobed (this is more clearly perceived from observing 
(a)

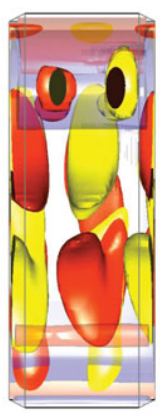

0

(b)

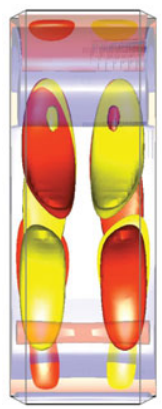

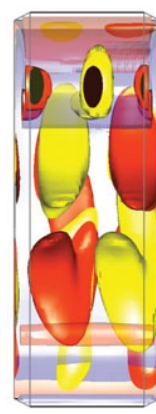

$\tau$

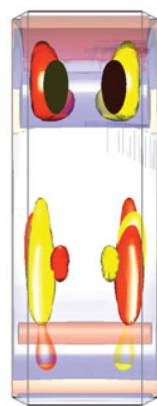

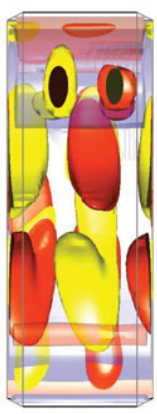

$2 \tau$

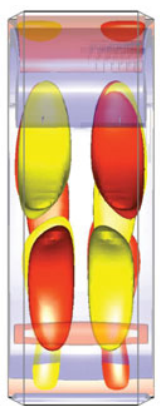

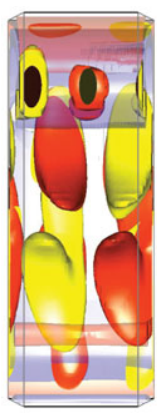

$3 \tau$

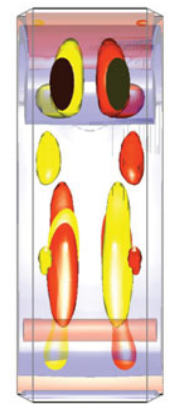

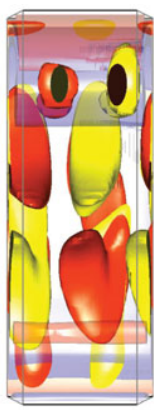

$4 \tau$

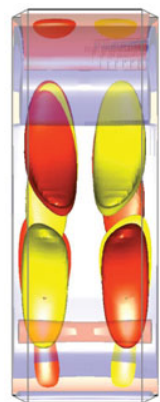

FIGURE 4. (Colour online) Isosurfaces of the quasi-periodic modes $(a)$ TW and $(b) \mathrm{SW}$, strobed at the wall oscillation period for $\Psi=4 / 3, k=33, R e=1200\left(1.7 \%\right.$ above $\left.R e_{c}\right)$. The view is taken outwards from the (vertically aligned) axis, and is of the subspace domain for $k=33$ (i.e. $2 \pi / 33$ in azimuthal extent, oriented horizontally). The rear wall is the oscillating wall. Translucent isosurfaces show azimuthal vorticity component, solid isosurfaces show radial vorticity component, with light and dark surfaces being of the same absolute value but of opposite sign. Animations are available as supplementary movies 2 and 3.

supplementary movie 2). The rate of precession is very close to the imaginary part of the Floquet exponent in these weakly nonlinear cases. For example, the corresponding linear analysis at this point in parameter space gives the secondary period required for the TW to traverse one wavelength as $\tau_{s}=4.08 \tau$, very similar to results obtained in the Cartesian case (see Blackburn \& Lopez 2003b, figure 12a), and indeed figure 4(a) shows that the wave has travelled just slightly less than one wavelength in four wall oscillation periods. The SW solution does not translate in azimuth, and while it has a reflection symmetry (about the central azimuthal coordinate), the flow pattern is always different at each multiple of $\tau$.

Figure 5 shows two snapshots of vorticity isosurfaces for a mode A solution, also for $\Psi=4 / 3$, but at higher $R e=1320$ and smaller $k=7$ than the mode QP case discussed above. These may be compared with a mode A solution for the Cartesian geometry shown in figure $9(b)$ of Blackburn \& Lopez $(2003 b)$; the features are qualitatively the same. Mode A is synchronous with the driven wall and does not involve any azimuthal precession. As in the Cartesian geometry, mode A has broken $H$-symmetry, but satisfies a new symmetry relationship

$$
(u, v, w)(r, \theta, z, t)=(u, v,-w)(r, \theta+\pi / k,-z, t+T / 2) .
$$


(a)

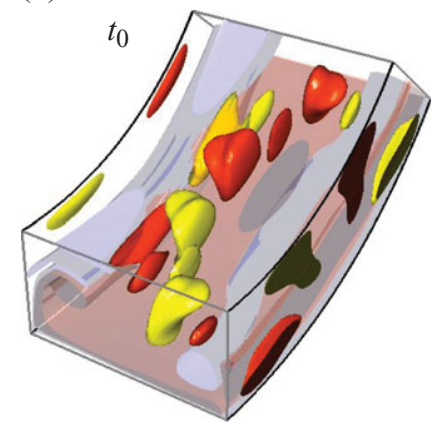

(b)

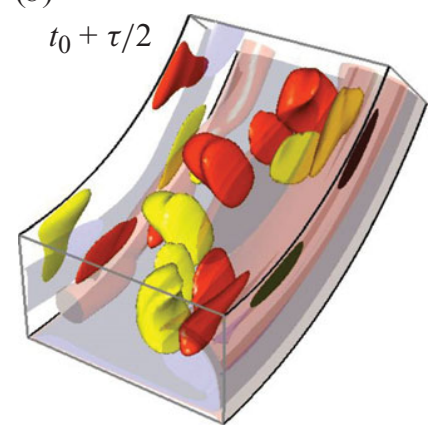

FIGURE 5. (Colour online) Isosurfaces, half a period apart, of mode A for a subspace solution with $\Psi=4 / 3, k=7, R e=1320\left(2.5 \%\right.$ above $\left.R e_{c}\right)$; translucent isosurfaces show azimuthal vorticity and solid isosurfaces show radial vorticity, with light and dark surfaces being of the same absolute value but of opposite sign. An animation is available as supplementary movie 4.

We note that for the cylinder wake, mode A retains $H$-symmetry and mode B breaks it - the opposite of what occurs in this cavity system. As explained in Blackburn \& Lopez (2003b), the naming convention for modes A and B was adopted for the Cartesian cavity based on the superficial visual similarity of the structure of instability modes with the circular cylinder case, but the linkage between mechanisms and loss of symmetry is apparently arbitrary and requires examination on a case-by-case basis.

\subsection{Weakly nonlinear dynamics in $k$-restricted subspaces}

The overall spatio-temporal structure of the three-dimensional modes does not change qualitatively with $\Psi$. Now, we address whether there are differences in their weakly nonlinear dynamics due to variations in $\Psi$, while retaining the restriction to single azimuthal wavenumber subspaces. The measure used to indicate bifurcation from the basic state is the solution kinetic energy contained in the azimuthal velocity component (which is zero in the base flow); its non-dimensional form is

$$
q_{\theta}=\frac{k}{8 \pi \Gamma} \int_{r_{i}}^{r_{0}} \int_{-\Gamma / 2}^{\Gamma / 2} \int_{-\pi / k}^{\pi / k}\left\langle v^{2}\right\rangle r \mathrm{~d} \theta \mathrm{d} z \mathrm{~d} r
$$

Figure 6 illustrates the nonlinear behaviour for the quasi-periodic modes TW and $\mathrm{SW}$, and the synchronous mode A, at $\Psi=4 / 3$ in subspaces restricted to the critical azimuthal wavenumber for each mode predicted by the Floquet analysis, $k=33$ for TW and SW, and $k=7$ for mode A. For the quasi-periodic modes, figure 6(a) indicates that both TW and SW solutions bifurcate supercritically from the basic state with $q_{\theta}$ growing from zero with $R e$, and that the TW solutions have larger $q_{\theta}$ than SW. This implies (Crawford \& Knobloch 1991) that TW will be stable to general perturbations, while SW will be unstable. Indeed, nonlinear computations show this to be the case, as was found with the Cartesian geometry as well as for quasi-periodic modes of circular and square cylinder wakes (Blackburn et al. 2005). For mode A, figure $6(b)$ shows the bifurcation to be subcritical, with a substantial hysteresis interval in $R e$, delimited by critical $R e$ for the Floquet instability of the base state to mode A, and the critical Re corresponding to the saddle-node bifurcation of stable and unstable mode A nonlinear states; this saddle-node critical $R e$ is only slightly larger than the critical $R e$ for mode QP.

Figure 7 shows the variation of $q_{\theta}$ with $R e$ for mode A at $(a) \Psi=0.9, k=15$ and (b) $\Psi=1.4, k=6$, i.e. one case with the inner and the other with the outer cylindrical wall 

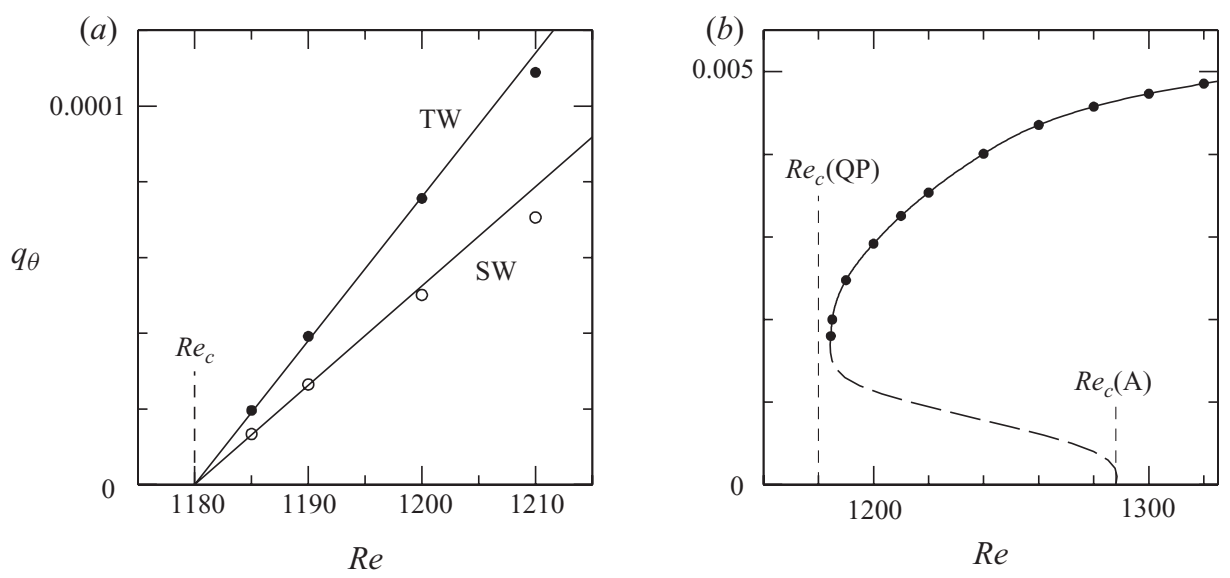

FIGURE 6. Variation of $q_{\theta}$ with $R e$ for $(a)$ TW and SW states restricted to the $k=33$ subspace, and $(b)$ mode A restricted to a $k=7$ subspace, both with $\Psi=4 / 3, S t=100$. The symbols on the solid curves indicate fully saturated computed nonlinear states and the long-dashed parts correspond to an estimate of an unstable branch. The vertical short-dashed lines identify critical Reynolds numbers for the various states, as labelled.
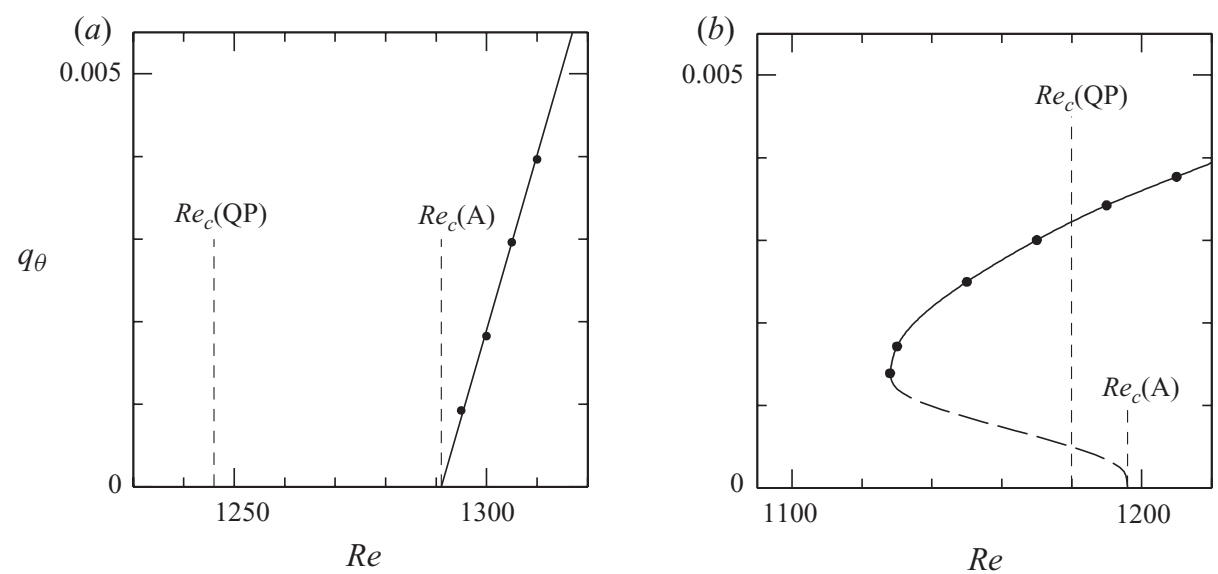

FIgURE 7. Variation of $q_{\theta}$ with $R e$ for mode A at (a) $\Psi=0.9$, restricted to the $k=15$ subspace, and $(b) \Psi=1.4$, restricted to the $k=6$ subspace. The symbols on the solid curves indicate fully saturated computed nonlinear states and the long-dashed parts correspond to an estimate of an unstable branch. The vertical short-dashed lines identify critical Reynolds numbers for the various states, as labelled; values for mode QP are shown for comparison.

oscillating. In each case the critical Re for mode A is greater than that for mode QP. For the driven inner wall case (figure 7a), mode A bifurcates supercritically, whereas for $\Psi=1.4$ (figure $7 b$ ) the bifurcation is subcritical, and the associated saddle-node bifurcation occurs at $R e$ lower than the critical $R e$ for mode QP. Generally, for $\Psi<1$ mode A has supercritical behaviour (first Landau constant positive), for $\Psi=1$ it is marginal (first Landau constant approximately zero), while for $\Psi>1$, the bifurcations to mode A become increasingly subcritical in nature (first Landau constant increasingly negative), with the associated hysteresis range of Re overlapping the critical $R e$ for mode QP. Of course, the nonlinear competition between modes A 
and QP under such conditions cannot be determined from $k$-restricted subspace simulations.

\section{Nonlinear mixed modes}

Nonlinear solutions computed in restricted subspaces are typical in numerical investigations of three-dimensional Floquet instabilities, where spanwise periodicity is assumed (e.g. Barkley \& Henderson 1996; Blackburn \& Lopez 2003b; Sheard, Thompson \& Hourigan 2004; Blackburn et al. 2005; Sheard et al. 2009). Especially in Cartesian geometries, this may seem a reasonable expedient since the spanwise spectrum is continuous in the infinite-geometry case, although examples in which this issue was tackled by expanding the extent of the discrete spectrum are provided by Henderson (1997) and Avila et al. (2007). In the present driven annular system, the azimuthal Fourier spectrum is discrete and simulations in a more complete space that includes low and high modes, but where resolution requirements in the $(r, z)$ plane are relatively undemanding, can be readily accommodated.

The simulations discussed in this section represent flows in the full closed annular geometry, and thus can be realized in a physical experiment. Our motivation was to examine quasi-periodic instabilities, which are of relatively modest spanwise/azimuthal module, and sufficient Fourier modes are included to resolve the most-amplified azimuthal wavenumber of the linear quasi-periodic instability at each radius ratio $\Psi$. Typically, we use $k_{\max }=4 k_{Q P}$ with $R e$ a few per cent above the critical value for linear instability of quasi-periodic modes, but well below the critical value for linear instability of mode A (and also of mode B). This gives a spatial resolution identical to that employed for the subspace calculations, but allows the larger azimuthal length scales associated with other modes to also be represented and resolved.

Flows have been examined for a range of radius ratios: $\Psi=9 / 10,10 / 9,5 / 4$ and $4 / 3$ (as well as $\Psi=1$ with the simulations restricted to a subspace of the continuous spectrum). Reporting of results concentrates on the case for $\Psi=4 / 3$, whose behaviour is representative of the others, and whose geometry is readily realizable in a physical experiment, since for given physical length scale $h$, it has the smallest circumference of the cases simulated. The initial conditions in each case were the TW state computed in the subspace simulation, to which is added about one part in $10^{4}$ of Gaussian white noise. Temporal evolution of energies in the azimuthal Fourier modes, given by

$$
E_{k}=\frac{1}{2 \Gamma} \int_{r_{i}}^{r_{0}} \int_{-\Gamma / 2}^{\Gamma / 2} \widehat{\boldsymbol{u}}_{k} \cdot \widehat{\boldsymbol{u}}_{k}^{*} r \mathrm{~d} z \mathrm{~d} r,
$$

where $\widehat{\boldsymbol{u}}_{k}$ is the Fourier transform of velocity for azimuthal mode $k$, are used as a diagnostic.

A typical evolution of Fourier modal energies for a full-annulus solution at $\Psi=4 / 3$ and $R e=1200\left(1.7 \%\right.$ above $\left.R e_{c}\right)$ is shown in figure 8 . The simulation has 132 azimuthal Fourier modes, i.e. 264 azimuthal planes of real data. At early times, the modal energies of the axisymmetric component, $E_{0}$, as well as for the leading wavenumber for the initiating TW solution and its azimuthal harmonics $\left(E_{33}, E_{66}, E_{99}\right)$ evolve at relatively constant levels, weakly modulated at the driving wall frequency. Within about 50 forcing periods, the perturbations have organized in the other modes and they start to grow exponentially. The Fourier modes with $k$ being linear combinations of 33 and 6 (other than $k=33$ and harmonics) are the fastest growing. Upon initial nonlinear saturation, after $t \approx 200 \tau$, there is a regular exchange of energy 


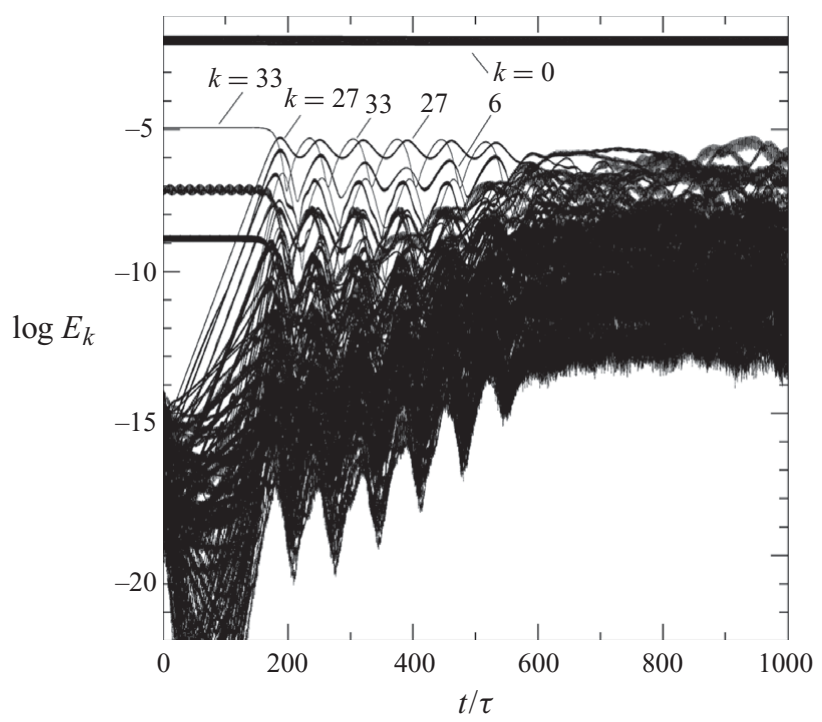

FIGURE 8. Evolution of Fourier modal energies for the full-annulus DNS at $\Psi=4 / 3$, $R e=1200$, starting at $t=0$ with a $k=33 \mathrm{TW}$ subspace solution to which a small amount of Gaussian white noise was added.

(a)

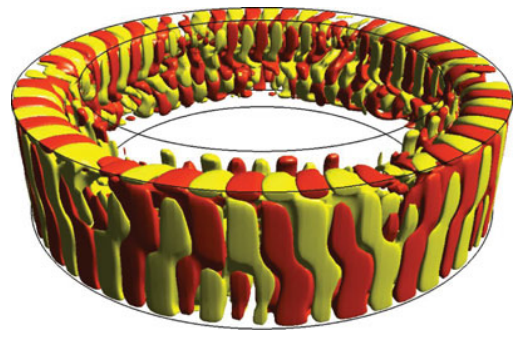

(b)

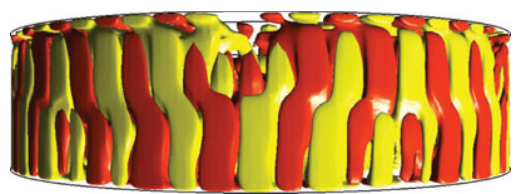

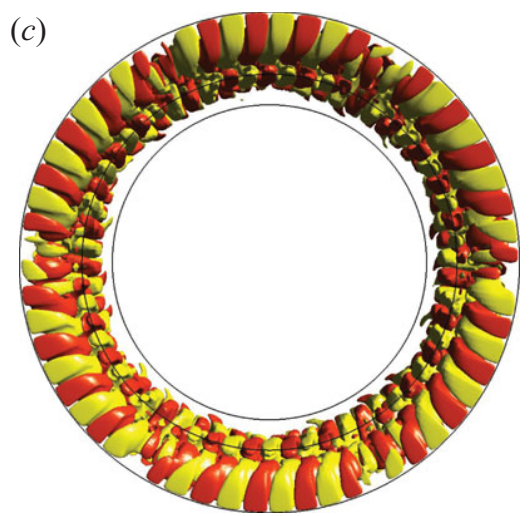

FIGURE 9. (Colour online) Snapshots of the mixed-mode state in the full annulus for $\Psi=4 / 3$, $R e=1200$ at $t / \tau=520$, showing isosurfaces of the azimuthal velocity component at levels $v= \pm 0.09$ in $(a)$ an oblique view, $(b)$ a radial view and $(c)$ an axial view. See also supplementary movies 5 and 6 .

between these modes, with a period of about $70 \tau$. This mixed-mode state persists until approximately $t / \tau=600$, by which time the remaining $E_{k}$ have saturated nonlinearly and the behaviour appears temporally chaotic.

The initial mixed-mode state is relatively simple to interpret and so its dynamics are considered before turning to the fully saturated state. Figure 9 shows azimuthal velocity isosurfaces, viewed from various angles, of the mixed-mode state at $\Psi=4 / 3$ and $R e=1200$, taken at time $t / \tau=520$ during the long-period quasiregular energy exchange regime between linear combinations of $k=6$ and $k=33$. Supplementary movies 5 and 6 provide animations of these isosurfaces which show the 
(a)

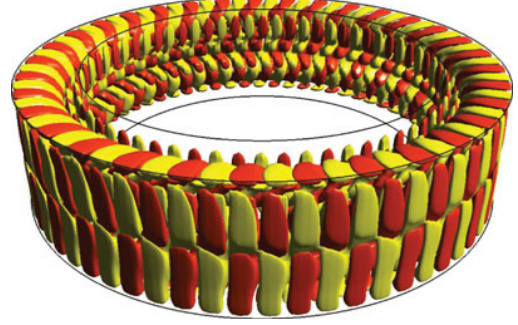

(b)
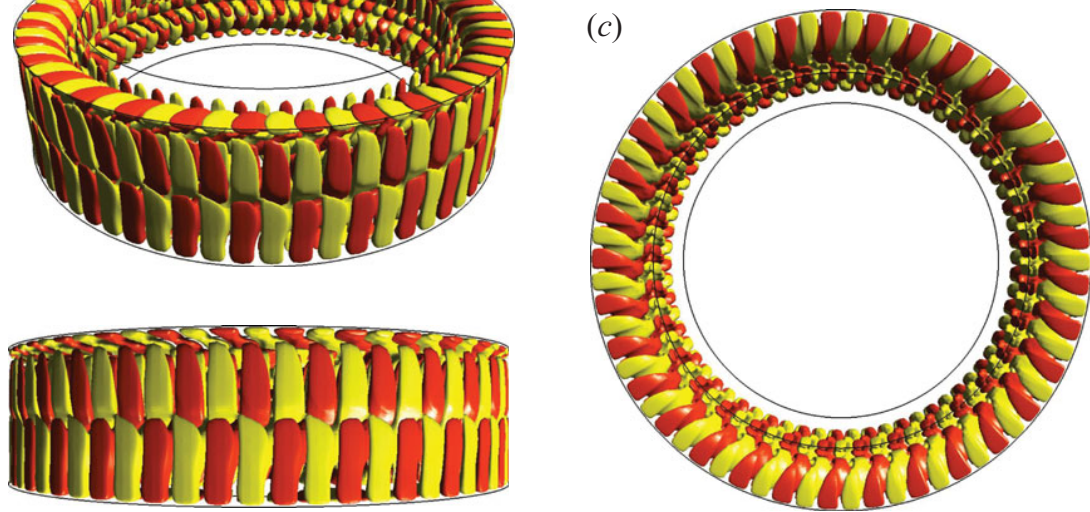

FIGURE 10. (Colour online) Snapshots of the mixed-mode state in the full annulus for $\Psi=4 / 3$, $R e=1200$ at $t / \tau=520$, as in figure 9, but filtered to show azimuthal Fourier mode $k=33$ and its harmonics. The isosurfaces are of the azimuthal velocity component at levels $v= \pm 0.09$ in $(a)$ an oblique view, $(b)$ a radial view and $(c)$ an axial view. See also supplementary movies 7 and 8 .

spatio-temporal behaviour over $140 \tau$, or two long-period cycles visible in figure 8 . When viewing the animations it should be borne in mind that these are strobed at the wall oscillation period and so much of the continuous temporal modulation that is visible in the subspace animations (supplementary movies 2-4) is suppressed strobing is used both to reduce the size of the animations and to emphasize travelling wave aspects.

First, note that the isosurfaces shown in figure 9 (and subsequent related figures) are of azimuthal velocity component, which is identically zero in the base state; the isosurfaces are representative of three-dimensional instability. Second, one will observe that the dominant structure present in figure 9 is a quasi-regular array of approximately 33 positive-negative interlinked isosurfaces; since the original TW structure at initiation is at $k=33$ these isosurfaces predominantly represent TW components. Third, the animations very clearly suggest an underlying TW state, e.g. the animation (supplementary movie 5) which corresponds to the view in figure $9(c)$ shows a counter-clockwise (CCW) sense of precession. Finally, note that in the view presented in figure 9(c), there are six azimuthal locations at which the regular TW structure appears to break down (one of these locations is central in the radial view shown in figure $9 b$ ); this six-fold irregularity is associated with instability mode A.

The modal energy time series of figure 8 show that in the early mixed-mode state (approximately for $t / \tau \in[200,600]$ ) the dominant components are formed by $k=6, k=33$, their harmonics, as well as linear combinations of the two, such as $k=27$, which is associated with their nonlinear interaction. Figures 10 and 11 are isosurfaces of the state shown in figure 9 filtered to show the contributions only in azimuthal modes $k=33$ and its harmonics, $k=33 n$, (figure 10) and azimuthal modes $k=6 n$ (figure 11). Supplementary movies 7 and 8 for $k=33 n$ show that these modal components behave very much like a pure TW, with a steady CCW precession when regarded from the viewpoint of figure 10(c), but the state's energy has an additional long-period $(\Delta t / \tau \approx 70)$ modulation associated with exchanges produced by the mixed-mode state. 
(a)

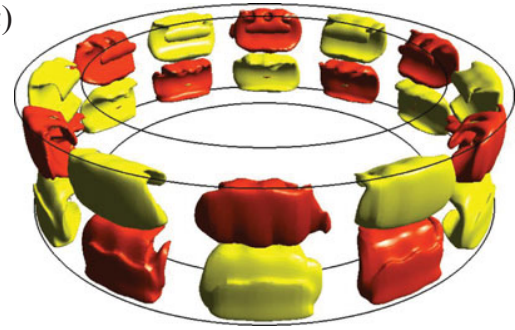

(b)

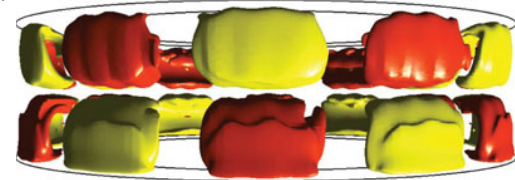

(c)

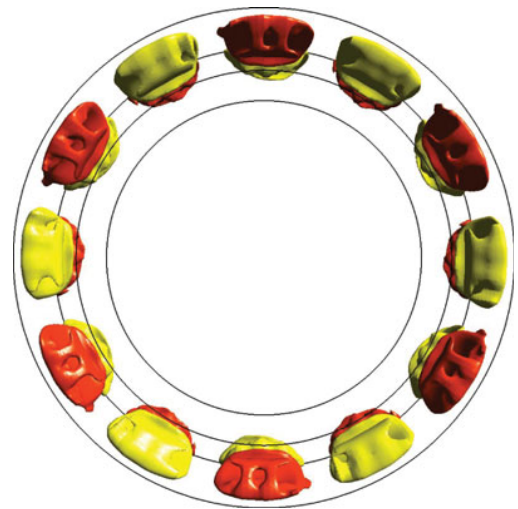

Figure 11. (Colour online) Snapshots of the mixed-mode state in the full annulus for $\Psi=4 / 3$, $R e=1200$ at $t / \tau=485$, filtered to show azimuthal mode $k=6$ and its harmonics. The isosurfaces are of the azimuthal velocity component at levels $v= \pm 0.09$ in $(a)$ an oblique view, (b) a radial view and $(c)$ an axial view. See also supplementary movies 9 and 10.

The $k=6 n$ components (figure 11, supplementary movies 9 and 10 ) have a character typical of mode A, again with a long-period modulation associated with mixedmode behaviour. However, these mode A-type structures no longer maintain a fixed azimuthal location, as they do in the linear analysis and the subspace calculations, but have a retrograde precession (CW) compared to the $k=33 n$ structures. From supplementary movies 9 and 10 , which extend over $\Delta t / \tau=140$, i.e. two longperiod modulations of energy, these structures are seen to precess $\Delta \theta \approx 2 \pi / 6$, or approximately one wavelength over this time interval. We note (see $\S 6.1$ ) that since a pure $k=33 \mathrm{TW}$ state takes approximately $4.08 \tau$ to traverse one azimuthal wavelength at $R e=1200, \Psi=4 / 3$, one TW module takes approximately $33 \times 4.08 \tau=135 \tau$ to traverse a complete circle. This is close to the long-period retrograde traverse time $(\sim 140 \tau)$ for one azimuthal module of the $k=6 n$ mode shown in figure 11, supplementary movies 9 and 10 .

After $t / \tau \approx 650$ in figure 8 , energies in all Fourier modes reach approximate statistical equilibrium, and long-period quasi-regular temporal structure is not evident. Figure 12 shows the time average of $E_{k}$ taken over $t / \tau \in[860,1000]$. There is a broad band of energy centred around $k=33$ the wavenumber of the initial TW state (albeit with two sub-peaks at $k=30$ and 35 ), as well as two other progressively weaker broad energy bands centred around $k=66$ and $k=99$ which can be considered harmonics of the $k \sim 33$ broad band. There is also a sharp peak at $k=6$ associated with a mode A-type instability, as noted above.

Figure 13 is representative of the fully saturated mixed-mode state. The spatial structure is again suggestive of an underlying mode QP TW state, but with less order than exists at earlier times (cf. figure 9). Animations (see supplementary movies 11 and 12) support this assessment: overall the appearance is of shortwavelength mode QP TW structures that on average appear to precess in the same sense $(\mathrm{CCW})$ as observed in supplementary movies 5 and 6 , although for some time intervals the apparent sense of motion in the (strobed) animations is retrograde.

The spatio-temporal behaviour of the other cases computed at $\Psi=9 / 10,10 / 9,5 / 4$ and (Cartesian) $\Psi=1$ was qualitatively similar to that of $\Psi=4 / 3$, discussed above. All cases were initiated with a saturated TW state computed in a subspace, projected 


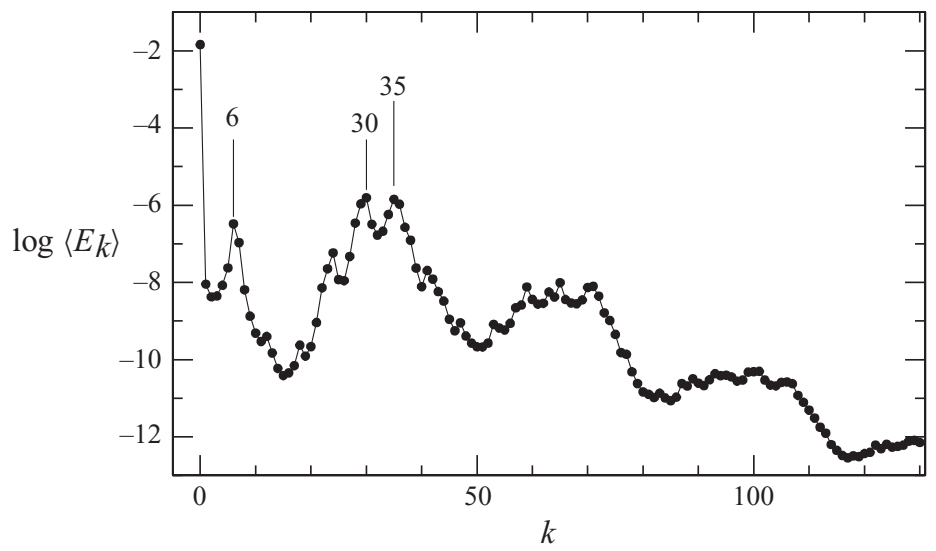

FiguRE 12. Time-average modal energies for the large-time end of the evolution shown in figure 8. Linear analysis predicts Fourier modes $k=6$ and $k=33$ are the most amplified wavenumbers for modes A and QP, respectively, though only mode QP is linearly unstable at $R e=1200$.

(a)

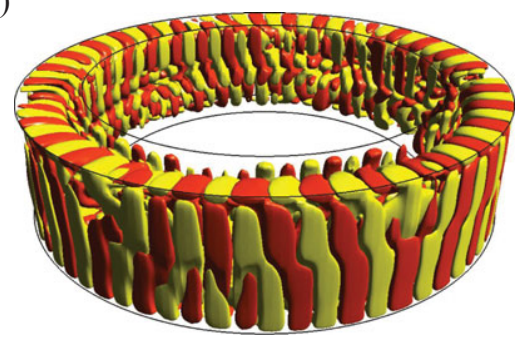

(b)

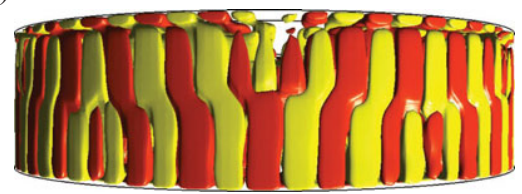

(c)

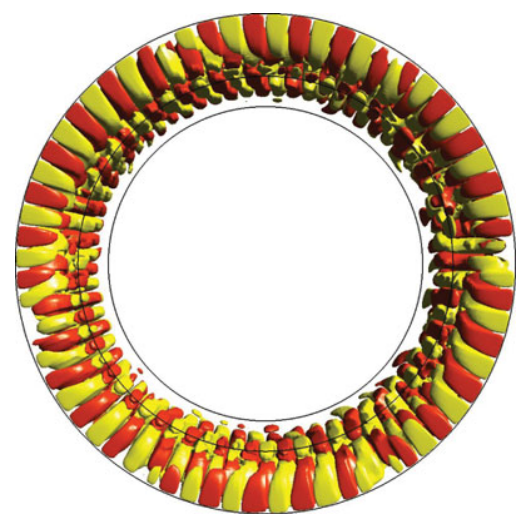

Figure 13. (Colour online) Snapshots of the mixed-mode state in the full annulus for $\Psi=4 / 3$, $R e=1200, t / \tau=860$ after full saturation has been reached; compare to figure 9 . The isosurfaces are of the azimuthal velocity component at levels $v= \pm 0.09$ in $(a)$ an oblique view, $(b)$ a radial view and $(c)$ an axial view. See also supplementary movies 11 and 12.

to a full annulus (or, for the $\Psi=1$, a periodic geometry with sufficient spanwise extent to accommodate both modes A and QP), to which a small amount of white noise was added. For all cases, energies in Fourier modes associated with instability mode A initially grew exponentially in time, reaching a preliminary saturation to a mixedmode state with mode QP and mode A components. Later, energies in all remaining Fourier modes continued to grow until a fully saturated and temporally erratic state was reached (i.e. similar behaviour to that shown in figure 8). An interesting feature for $\Psi=10 / 9$ is that according to linear analysis (figure 2 ) the critical $R e$ for mode A, 
$R e_{c}>1500$, is substantially greater than that for mode $\mathrm{QP}, R e_{c}=1192$. One might reasonably expect a pure TW state to be maintained in the full-annulus simulation at $2.5 \%$ above criticality, $R e=1223$. However, nonlinear effects excite mode A behaviour and the result is a mixed mode with complicated spatio-temporal dynamics. It is of interest to note that the wavenumber observed for mode A $(k=15)$ in this case is very close to the value predicted by (5.1), which with the Cartesian value $\lambda=3.81$, gives $k=15.7$.

\section{Discussion and conclusions}

We have examined instabilities in a system with a base state that has $Z_{2} \times$ $O(2)$ symmetry characteristic of the infinite von Kármán vortex street, but which is physically realizable without end effects. The system consists of a liquid-filled annulus with either the inner or outer cylinder oscillating in the axial direction. There are four control parameters: the aspect ratio of the annular gap (kept fixed at 2); the radius ratio $\Psi$ that measures the degree of curvature and is varied (the Cartesian case with $\Psi=1$ was analysed in detail in Blackburn \& Lopez 2003b); and amplitude and frequency of the oscillations, measured by the Reynolds and Stokes numbers. The main motivation for the present investigation was to investigate modulated travelling waves, which are a generic instability in systems with $Z_{2} \times O(2)$ spatio-temporal symmetry. In this non-autonomous $Z_{2} \times O(2)$ symmetric system, having at least two control parameters to vary allows access to regions of parameter space, where any of the three instabilities to three-dimensional flow are the primary bifurcation from the basic state (Marques et al. 2004). This is in contrast to autonomous systems such as bluff body wakes, where there is only a single dynamic control parameter. In the present work, the Stokes number was fixed so that for the given geometry, quasiperiodic modes (either TW or SW) are the first to bifurcate from the the axisymmetric time-periodic basic state. The variation in curvature has a definite, but subtle effect on the observed instabilities. For moderate curvature, with $\Psi \in[0.85,1.45]$, quasiperiodic modes are the first to bifurcate from the basic state with increasing $R e$ at $S t=100$. For larger curvature, longer wavelength type-A modes are first to bifurcate. The azimuthal wavelengths of the least-stable modes of type A and QP are distinct, and remain close to the values obtained in the Cartesian geometry for the range of $\Psi$ considered.

Observations made in physical experiments with a related, but finite-length Cartesian cavity (Leung et al. 2005), have shown quasi-periodic states with frequencies and spatial structure very close to those of the predicted TW mode, but with no net propagation in the spanwise direction, presumably owing to the presence of endwalls. In the annular problem, the spatio-temporal dynamics are continuous with $\Psi$ varying across the Cartesian limit. This would suggest that differences in the dynamics between the finite Cartesian cavity and a very low curvature annular cavity would be due to endwall effects.

The weakly nonlinear dynamics of the least stable wavenumber modes of type A and QP were first examined in subspaces of restricted azimuthal periodicity, selected on the basis of the linear analysis. Numerical simulations in wavelength-restricted subspaces have been a typical expedient in previous investigations (Barkley \& Henderson 1996; Henderson \& Barkley 1996; Blackburn \& Lopez 2003b; Sheard, Thompson \& Hourigan 2004), in part owing to the significant cost of full-space DNS especially near $\Psi=1$. In such restricted subspaces, mode QP bifurcates supercritically 
at all considered values of $\Psi$, and modulated TW states of this mode are stable, while modulated SW are unstable. On the other hand, depending on the curvature and which wall oscillates, mode A can bifurcate either supercritically (for $\Psi<1$ ) or subcritically $(\Psi>1)$, with the Cartesian case, $\Psi=1$, being marginal with a first Landau constant of approximately zero, as earlier established by Blackburn \& Lopez $(2003 b)$.

In the present problem, subspace-restricted nonlinear results are, however, not robust. Simulations using well-resolved DNS in the full domain show that the nonlinear dynamics, even in parameter regimes very close to primary instabilities, are not well predicted by either linear analysis or nonlinear computations in subspaces restricted to the fastest growing linear modes. The nonlinear dynamics in the full driven cavity, when initiated with a TW mode from a subspace computation, show a long transient during which a mode A component grows, saturates and interacts nonlinearly with the TW mode, resulting in mixed-mode dynamics which persist for a few hundred forcing periods. This simple mixed-mode state is ultimately unstable as the other Fourier modes grow, saturate and render the mixed mode with complicated spatio-temporal behaviour. Despite the lack of robustness in detail, the final state still retains the modulated TW as a readily identifiable dominant component. This final state with a broad spectrum of energy across Fourier modes would also be observed in a physical experiment, where the complicated behaviour might be attributed to experimental noise or imprecision. Here, it is shown to be an inherent characteristic of the full nonlinear problem.

The type of behaviour described above, complicated spatio-temporal dynamics developing virtually directly from the basic state, has been observed in other flows, both experimentally and numerically. For example, in periodically modulated TaylorCouette flow (Marques \& Lopez 1997, 2000; Weisberg, Kevrekidis \& Smits 1997), Sinha, Kevrekidis \& Smits (2006) reported on experimental results that indicated a direct transition from the periodic axisymmetric basic state to complicated spatiotemporal flow in regimes where linear Floquet analysis and nonlinear subspacerestricted simulations predicted pure mode solutions, but full nonlinear simulations revealed that the complex spatio-temporal behaviour was a result of a cascade of symmetry-breaking bifurcations in an extremely narrow range of Reynolds numbers, much finer than could be resolved in the experiments (Avila et al. 2007). Similar cascades to spatio-temporal complexity have been found in rotating thermal convection, where the onset of Küppers-Lortz dynamics is reported to be a 'direct' transition from the basic state in experiments (Bodenschatz, Pesch \& Ahlers 2000), whereas full DNS shows that the complex dynamics also result from a cascade of symmetry-breaking bifurcations over an extremely narrow range of parameter space (Rubio, Lopez \& Marques 2010).

Inherent in the complex spatio-temporal dynamics in the driven annular cavity problem are the mixed-mode states. In the related $Z_{2} \times O(2)$ circular wakes flows, both physical experiments (Williamson 1996) and DNS studies (Henderson 1997) show the presence of $\mathrm{A}-\mathrm{B}$ mixed-mode behaviour at Reynolds numbers between the linear onset of modes A and B, and Barkley, Tuckerman \& Golubitsky (2000) have provided a normal form analysis of this mixed-mode interaction between the two synchronous states. In the annular driven cavity, however, the interaction is not between two synchronous states but rather between a synchronous mode $\mathrm{A}$ and a quasi-periodic mode TW. The theoretical analysis of such mode interactions (between a Floquet multiplier equal to one and a pair of complex-conjugate Floquet multipliers) 
is complicated and incomplete (Kuznetsov 2004). The results presented here provide insight into the associated dynamics in a physically realizable flow.

This work was supported through Merit Allocation Scheme Grant D77 from the Australian National Computational Infrastructure, the Monash University Small Grants Scheme, the US National Science Foundation and the Korean Science and Engineering Foundation WCU grant R32-2009-000-20021-0. We thank F. Marques for his many illuminating contributions throughout our pursuit of this area of research.

Supplementary movies are available at journals.cambridge.org/flm.

\section{REFERENCES}

Avila, M., Marques, F., Lopez, J. M. \& Meseguer, A. 2007 Stability control and catastrophic transition in a forced Taylor-Couette system. J. Fluid Mech. 590, 471-496.

Barkley, D., Blackburn, H. M. \& Sherwin, S. J. 2008 Direct optimal growth analysis for timesteppers. Intl J. Numer. Meth. Fluids 57, 1437-1458.

BARKLey, D. \& Henderson, R. D. 1996 Three-dimensional Floquet stability analysis of the wake of a circular cylinder. J. Fluid Mech. 322, 215-241.

Barkley, D., Tuckerman, L. S. \& Golubitsky, M. 2000 Bifurcation theory for three-dimensional flow in the wake of a circular cylinder. Phys. Rev. E 61, 5247-5252.

Blackburn, H. M. 2002 Three-dimensional instability and state selection in an oscillatory axisymmetric swirling flow. Phys. Fluids 14 (11), 3983-3996.

Blackburn, H. M. \& Lopez, J. M. 2002 Modulated rotating waves in an enclosed swirling flow. $J$. Fluid Mech. 465, 33-58.

Blackburn, H. M. \& LOPEZ, J. M. $2003 a$ On three-dimensional quasi-periodic Floquet instabilities of two-dimensional bluff body wakes. Phys. Fluids 15 (8), L57-L60.

Blackburn, H. M. \& LopeZ, J. M. $2003 b$ The onset of three-dimensional standing and modulated travelling waves in a periodically driven cavity flow. J. Fluid Mech. 497, 289-317.

Blackburn, H. M., Marques, F. \& Lopez, J. M. 2005 Symmetry breaking of two-dimensional time-periodic wakes. J. Fluid Mech. 522, 395-411.

Blackburn, H. M. \& Sheard, G. J. 2010 On quasiperiodic and subharmonic Floquet wake instabilities. Phys. Fluids 22, 031701-1-4.

Blackburn, H. M. \& Sherwin, S. J. 2004 Formulation of a Galerkin spectral element-Fourier method for three-dimensional incompressible flows in cylindrical geometries. J. Comput. Phys. 197 (2), 759-778.

Bodenschatz, E., Pesch, W. \& Ahlers, G. 2000 Recent developments in Rayleigh-Bénard convection. Annu. Rev. Fluid Mech. 32, 709-778.

Carmo, B. S., Sherwin, S. J., Bearman, P. W. \& Willden, R. H. J. 2008 Wake transition in flow around two circular cylinders in staggered arrangements. J. Fluid Mech. 597, 1-29.

Crawford, J. D. \& KNobloch, E. 1991 Symmetry and symmetry-breaking bifurcations in fluid dynamics. Annu. Rev. Fluid Mech. 23, 341-387.

Cross, M. C. \& Hohenberg, P. C. 1993 Pattern formation outside of equilibrium. Rev. Mod. Phys. $\mathbf{6 5}, 851-1112$.

Henderson, R. D. 1997 Nonlinear dynamics and pattern formation in three-dimensional wakes. J. Fluid Mech. 352, 65-112.

Henderson, R. D. \& BARKLey, D. 1996 Secondary instability in the wake of a circular cylinder. Phys. Fluids 8 (6), 1683-1685.

Julien, S., Lasheras, J. \& Chomaz, J.-M. 2003 Three-dimensional instability and vorticity patterns in the wake of a flat plate. J. Fluid Mech. 479, 155-189.

Kuznetsov, Y. A. 2004 Elements of Applied Bifurcation Theory, 3rd edn. Springer.

Lasheras, J. C. \& Meiburg, E. 1990 Three-dimensional vorticity modes in the wake of a flat plate. Phys. Fluids A 5, 371-380.

Leung, J. J. F., Hirsa, A., Blackburn, H. M., Lopez, J. M. \& Marques, F. 2005 Three-dimensional modes in a periodically driven elongated cavity. Phys. Rev. E 71, 026305-1-7. 
Leweke, T. \& Provansal, M. 1995 The flow behind rings: bluff body wakes without end effects. J. Fluid Mech. 288, 265-310.

Marques, F. \& Lopez, J. M. 1997 Taylor-Couette flow with axial oscillations of the inner cylinder: Floquet analysis of the basic flow. J. Fluid Mech. 348, 153-175.

Marques, F. \& Lopez, J. M. 2000 Spatial and temporal resonances in a periodically forced extended system. Physica D 136, 340-352.

Marques, F., Lopez, J. M. \& Blackburn, H. M. 2004 Bifurcations in systems with $Z_{2}$ spatiotemporal and $O(2)$ spatial symmetry. Physica D 189 (3-4), 247-276.

Meiburg, E. \& Lasheras, J. C. 1988 Experimental and numerical investigation of the threedimensional transition in plane wakes. J. Fluid Mech. 190, 1-37.

Rubio, A., Lopez, J. M. \& Marques, F. 2010 Onset of Küppers-Lortz-like dynamics in finite rotating thermal convection. J. Fluid Mech. 644, 337-357.

Sheard, G. J., FitzGerald, M. J. \& Ryan, K. 2009 Cylinders with square cross-section: wake instabilities with incidence angle variation. J. Fluid Mech. 630, 43-69.

Sheard, G. J., Thompson, M. C. \& Hourigan, K. 2003 From spheres to circular cylinders: the stability and flow structures of bluff ring wakes. J. Fluid Mech. 492, 147-180.

Sheard, G. J., Thompson, M. C. \& Hourigan, K. 2004 From spheres to circular cylinders: non-axisymmetric transitions in the flow past rings. J. Fluid Mech. 506, 45-78.

Sinha, M., Kevrekidis, I. G. \& Smits, A. J. 2006 Experimental study of a Neimark-Sacker bifurcation in axially forced Taylor-Couette flow. J. Fluid Mech. 558, 1-32.

Swift, J. W. \& WiesENFELD, K. 1984 Suppression of period doubling in symmetric systems. Phys. Rev. Lett. 52 (9), 705-708.

Tuckerman, L. S. \& Barkley, D. 2000 Bifurcation analysis for time steppers. In Numerical Methods for Bifurcation Problems and Large-Scale Dynamical Systems (ed. E. Doedel \& L. S. Tuckerman), pp. 453-566. Springer.

Vogel, M. J., Hirsa, A. H. \& Lopez, J. M. 2003 Spatio-temporal dynamics of a periodically driven cavity flow. J. Fluid Mech. 478, 197-226.

Weisberg, A. Y., Kevrekidis, I. G. \& SMiTs, A. J. 1997 Delaying transition in Taylor-Couette flow with axial motion of the inner cylinder. J. Fluid Mech. 348, 141-151.

Williamson, C. H. K. 1989 Oblique and parallel modes of vortex shedding in the wake of a circular cylinder at low Reynolds numbers. J. Fluid Mech. 206, 579-627.

Williamson, C. H. K. 1996 Three-dimensional wake transition. J. Fluid Mech. 328, 345-407. 